\title{
Parâmetros bioquímicos e cinética de lactato sanguíneo de sujeitos HIV+ submetidos ao treinamento físico combinado
}

CDD. 20.ed. 616.979201

796.073

http://dx.doi.org/10.1590/1807-55092015000400519

\author{
Joice Cristina dos Santos TROMBETA* \\ Roberto Carlos VIEIRA J UNIOR ${ }^{* *}$ \\ Túlio Augusto Bonfim FERNANDES* \\ Cássio Charnoski RUBIM* \\ J onato PRESTES \\ Fabrício Azevedo VOLTARELLI* ${ }^{*}$
}

*Faculdade de Educação Física, Universidade Federal de Mato Grosso.

**Universidade do Estado de Mato Grosso.

***Universidade Católica de Brasília.

\begin{abstract}
Resumo
0 objetivo deste estudo foi verificar a cinética de lactato sanguíneo (CLS) e a sua resposta ao treinamento físico combinado (TFC) bem como avaliar os efeitos sobre os aspectos bioquímicos, imunológicos, cardiorrespiratório e composição corporal de pessoas vivendo com HIV/AIDS. Doze voluntários HIV+ realizaram o TFC, por um período de 16 semanas, e foram submetidos às avaliações nos momentos pré, oito e 16 semanas de TFC. Ao final, houve um aumento das células TCD4+ e manutenção da carga viral, aumento do consumo máximo de oxigênio e da força muscular e, ainda, aumento do HDL-c e diminuição dos triglicerídeos e glicemia de jejum. Ainda, ocorreu aumento da CLS após o período de treinamento. Concluímos que o TFC influenciou positivamente os parâmetros imunológicos, bioquímicos, cardiorrespiratório e muscular, no entanto, a questão relacionada à CLS necessita de estudos futuros para maiores esclarecimentos.
\end{abstract}

Palavras-chave: HIV/AIDS; Cinética de lactato; Aptidão física, Força muscular; Sistema imune.

\section{Introdução}

O vírus HIV (vírus da imunodeficiência humana adquirida) é um membro da família "Retroviridae", sub-família Lentiviradae, sendo ele responsável por efeitos citopáticos à curto prazo e por uma infecção persistente que culmina em quadro clínico final conhecido como AIDS ("Acquired Immune Deficiency Syndrome"), que predispóe o indivíduo ao desenvolvimento de doenças oportunistas que, se não tratadas, o levam ao óbito ${ }^{1-3}$. Há alguns anos, receber o diagnóstico positivo era considerada uma sentença de morte, mas, atualmente, a AIDS é considerada uma doença de perfil crônico sem cura, mas há tratamento ${ }^{4}$.

A principal estratégia farmacológica empregada no gerenciamento do quadro de infecção causado pelo HIV/AIDS é a terapia antirretroviral de alta atividade (HAART, do inglês "Highly Active Antiretroviral Therapy"). A HAART inclui classes de drogas compostas por inibidores de transcriptase reversa análogos de nucleosídeos (ITRN), inibidores de transcriptase reversa não análogos de nucleosídeos
(ITRNN), inibidores de protease (IP), inibidores de fusão e, ainda, inibidores de integrase $e^{3-4}$.

Muito embora tenha possibilitado a reconstituiçấo do sistema imunológico e o aumento da sobrevida das pessoas vivendo com HIV/AIDS, o uso prolongado da HAART, assim como a infecção pelo vírus HIV, causam alteraçóes negativas e deletérias ao organismo desses sujeitos, tais como: complicaçóes cardiorrespiratórias (diminuição do consumo de oxigênio), musculares (sarcopenia e diminuição da força muscular), psiquiátricas (depressão), alteraçóes no perfil bioquímico (redução nos níveis de HDL-c e aumentos de LDL-c e glicose), dentre outras. Essas alteraçôes, quando analisadas separadamente, merecem atenção e, quando agrupadas, levam o organismo a desenvolver problemas cardiovasculares graves ${ }^{5-9}$.

Dentre as alteraçóes que pioram a qualidade de vida desses pacientes está a toxicidade mitocondrial de diversos tecidos, entre eles o hepático e o muscular, a qual leva 
à sua disfunção, fato que contribui substancialmente para a ocorrência de hiperlactacidemia de repouso ${ }^{10-11}$.

Intervençôes não-medicamentosas vêm sendo aplicadas para evitar/minimizar as consequências negativas advindas da associação entre a infecção do vírus HIV e o uso da HAART; dentre elas está a adesão ao programa de exercícios físicos regulares ${ }^{2,5,8-10,12}$. Diversos estudos são encontrados na literatura mostrando os benefícios advindos do treinamento físico de força e aeróbio, tais como: aumento e manutenção do consumo máximo de oxigênio, adaptações neurais, aumento da massa e força muscular bem como redução do percentual de gordura, melhora nos perfis lipídico e glicêmico, diminuição do risco coronariano, síntese de novas mitocôndrias e melhor vascularização (angiogênese), além da manutenção da carga viral e o aumento das células TCD4 $+^{5,9,13-14}$. No presente estudo, o intuito foi obter os efeitos desejados nas variáveis referentes aos treinamentos resistido e aeróbio em um curto espaço de tempo e, para isso, associou-se em uma mesma sessão de treino os dois componentes, sendo este conhecido como treinamento físico combinado (TFC).

Porém, há poucos relatos relacionados ao efeito crônico do TFC sobre os parâmetros imunológico, cardiorrespiratório, força muscular, assim como, sobre as elevadas concentraçôes de lactato sanguíneo em repouso em pessoas vivendo com HIV/AIDS, bem como sobre a cinética desse substrato durante

\section{Método}

Este estudo foi realizado na Universidade Federal de Mato Grosso (UFMT/ Cuiabá/MT) e contou com a participação de enfermeiros, médicos, professores de educação física e nutricionistas. O trabalho foi desenvolvido entre os meses de outubro de 2012 e julho de 2013, dando continuidade aos trabalhos desenvolvidos pelo grupo de pesquisa FIBAMEF/ NAFIMES sobre esta temática. O projeto de pesquisa foi aprovado pelo Comitê de Ética em Pesquisa do HUJM (n. protocolo: 673/09).

\section{Amostra}

A amostra foi composta por 12 pacientes HIV+ (Idade: 39,75 $\pm 10,67$ anos; tempo de portador: 7,75 \pm 7,88 anos; tempo de HAART: 6,41 \pm 5,93 anos) em tratamento no Serviço de Assistência o exercício nessa população. Em relação, ao lactato sanguíneo, um dos poucos trabalhos encontrados é o de BAUER et al. ${ }^{15}$, o qual investigou a cinética de lactato sanguíneo em pessoas HIV+ e sem a infecção, fazendo uso ou não da medicaçáo, após sessão aguda de exercício físico; esses autores observaram diferença no metabolismo do lactato entre os grupos analisados após a sessão aguda. A pesquisa de BAUER et al..$^{15}$, assim como os trabalhos de Roge et al. ${ }^{16}$, JoHn et al. ${ }^{17}$ e Mamiafo et al. ${ }^{18}$, analisaram agudamente e confirmaram a prevalência da hiperlactacidemia nessa população, reforçando a necessidade da realização de estudos envolvendo o efeito crônico do treinamento físico em pessoas vivendo com HIV/AIDS.

Sendo assim, a hipótese de nosso estudo é que ocorram efeitos positivos sobre as variáveis metabólicas relacionadas à saúde (parâmetros bioquímicos, imunológicos e antropométricos) e sobre a concentração de lactato de repouso (e também em exercício e durante o período de recuperação; i.e., cinética) em pessoas vivendo com HIV/AIDS submetidas ao TFC. Portanto, a relevância deste estudo consiste na possibilidade de proporcionar subsídios teóricos sobre o TFC em pessoas vivendo com HIV/AIDS, confirmando a importância do conhecimento sobre a infecção pelo HIV, as limitaçóes, os benefícios crônicos e a periodizaçáo do treinamento para este grupo especial, garantindo, assim, o desenvolvimento de intervençóes qualificadas.

Especializada (SAE) e Centro Estadual de Referência de Alta e Média Complexidade (CERMAC), ambos localizados no município de Cuiabá-MT, os quais finalizaram todas as etapas relacionadas às avaliaçōes, coletas e ao treinamento físico.

Os critérios de inclusão do estudo foram: ter idade mínima de 18 anos, ser sedentário, não ter nenhum impedimento quanto à prática de atividade física, estar fazendo uso da HAART, ter completado todas as etapas pré-treinamento e possuir frequência mínima de $85 \%$ nos treinamentos realizados. Não foram incluídos os pacientes que apresentaram: doenças inflamatórias agudas ou crônicas (artrites, lesôes músculo-articulares); doenças cardíacas e vasculares; câncer; contagem de células TCD4+ menor que 200 células $/ \mathrm{mm}^{3}$; gravidez e que não obtiveram liberação médica. 


\section{Procedimentos experimentais}

\section{Adaptação ao protocolo de treinamento físico}

Com o intuito de familiarizar os participantes ao treinamento físico, foram realizadas duas semanas de adaptação. Para o treinamento físico resistido, o participante foi orientado a realizar uma única série de 12 repetiçóes, com a carga mínima, nos seguintes aparelhos: $1 / 2$ agachamento na barra guiada, supino reto articulado, cadeira flexora de joelhos, puxada alta à frente, "leg press" $45^{\circ}$, tríceps na polia, desenvolvimento na máquina, flexão plantar e rosca direta. Nesta fase, foi utilizada a escala perceptiva de esforço imediatamente após a realização do movimento em cada equipamento; pediu-se ao participante que o mesmo apontasse na escala o valor correspondente a sensaçáo percebida do esforço realizado. Dependendo do valor apontado (6 a 20), a carga no respectivo aparelho sofria modificaçóes para a próxima aula. Para o fortalecimento da regiáo abdominal, os participantes realizaram exercícios para os músculos abdominais, alternadamente, a cada três aparelhos de musculação. Neste primeiro momento, apenas foram orientados quanto à técnica de execução e realizaram entre 10 a 20 exercícios abdominais.

Logo em seguida, os sujeitos efetuaram uma caminhada orientada (treinamento aeróbio) de no mínimo 20 minutos na primeira semana e 30 minutos a partir da segunda semana, a qual foi realizada na pista de atletismo da UFMT. Orientou-se que cada participante observasse o número de voltas completadas em cada dia de treino. Os alongamentos foram realizados antes e após o treinamento.

\section{Treinamento físico combinado - TFC}

O período total do programa de TFC foi de 18 semanas, composto da seguinte maneira: duas semanas de adaptação e 16 semanas de treinamento físico propriamente dito. As atividades relacionadas ao processo de treinamento físico foram divididas da seguinte maneira: aquecimento - constituído de alongamentos para o corpo todo; parte principal - TFC; volta à calma - realização de alongamentos para relaxamento. Ao todo o treinamento teve a duraçáo de 70 a 80 minutos (30 minutos de treinamento físico resistido, 30 a $40 \mathrm{mi}-$ nutos de aeróbio e 10 minutos de alongamentos) com frequência de três vezes na semana (dias intercalados).

$\mathrm{O}$ treinamento físico resistido foi realizado na forma de circuito, onde os participantes realizaram uma série em cada equipamento de musculação (descritos na adaptação) e a cada três equipamentos era realizada uma sequência de abdominais (variando de 10 a 30, dependendo da capacidade de cada sujeito). Ao final do circuito, foi realizado um intervalo de recuperação de aproximadamente 90 segundos. Posteriormente, repetiu-se a mesma sequência. Os valores de carga foram obtidos a partir do teste de $10 \mathrm{RM}$ e os exercícios abdominais e de flexão plantar através do teste de resistência muscular localizada (número máximo de execuçóes por minuto). A periodização do treinamento físico resistido está descrita na TABELA 1.

TABELA 1 - Periodização do treinamento resistido durante as 16 semanas.

\begin{tabular}{|c|c|c|c|c|}
\hline Semanas & $1^{a}$ e $2^{a}$ & $3^{a}$ e $4^{a}$ & $5^{a}$ e $6^{a}$ & $7^{a}$ e $8^{a}$ \\
\hline N. de voltas & 2 & 2 & 2 & 2 \\
\hline Séries & 1 & 1 & 1 & 1 \\
\hline Zona de treinamento & 12 a 15 & 10 a 12 & 8 a 6 & 4 a 6 \\
\hline Carga (10 RM) & $60 \%$ & $75 \%$ & $85 \%$ & $100 \%$ \\
\hline Semanas & $9^{a}$ e $10^{a}$ & $11^{\mathrm{a}} \mathrm{e} 12^{\mathrm{a}}$ & $13^{\mathrm{a}}$ e $14^{\mathrm{a}}$ & $15^{\mathrm{a}}$ e $16^{\mathrm{a}}$ \\
\hline N. de voltas & 2 & 2 & 2 & 2 \\
\hline Séries & 1 & 1 & 1 & 1 \\
\hline Zona de treinamento & 12 a 15 & 10 a 12 & 8 a 6 & 4 a 6 \\
\hline Carga (10 RM) & $60 \%$ & $75 \%$ & $85 \%$ & $100 \%$ \\
\hline
\end{tabular}

Após a realização do treinamento físico resistido, os sujeitos iniciavam o treinamento físico aeróbio, consistido de caminhada em pista de atletismo ( 400 metros) de 30 a 40 minutos; esse treinamento foi prescrito com base nos valores da frequência cardíaca individual obtidos pela seguinte fórmula: FCmáxima $=208-(0,7 \times \text { idade })^{9}$; durante toda o período de treinamento físico, os participantes fizeram uso de frequencímetro $\left(\mathrm{POLAR}^{\circledR}\right)$. A periodização do treinamento físico aeróbio encontra-se na TABELA 2. 
TABELA 2 - Periodização do treinamento aeróbio durante as 16 semanas.

\begin{tabular}{|c|c|c|c|c|}
\hline Semanas & $1^{a}$ e $2^{a}$ & $3^{a}$ e $4^{a}$ & $5^{a}+6^{a}$ & $7^{a}$ e $8^{a}$ \\
\hline Carga (FCmáx) & 60 a $70 \%$ & 60 a $70 \%$ & 60 a $70 \%$ & 60 a $70 \%$ \\
\hline Duração (min) & 30 & 30 & 40 & 40 \\
\hline Semanas & $9^{a}$ e $10^{a}$ & $11^{\mathrm{a}}$ e $12^{\mathrm{a}}$ & $13^{\mathrm{a}}$ e $14^{\mathrm{a}}$ & $15^{\mathrm{a}}$ e $16^{\mathrm{a}}$ \\
\hline Carga (FCmáx) & 70 a $80 \%$ & 70 a $80 \%$ & 70 a $80 \%$ & 70 a $80 \%$ \\
\hline Duração (min) & 30 & 30 & 40 & 40 \\
\hline
\end{tabular}

\section{Testes e exames}

\section{Exames bioquímicos}

As coletas sanguíneas foram realizadas por enfermeiras experientes cedidas pelo CERMAC-MT. As variáveis analisadas foram as seguintes: Aspectos imunológicos: Quantificação da carga viral (método: b-DNA Kit: HIV 3.0 RNA); Contagem das células TCD4+, células TCD8+ e células CD45+; Razão TCD4+/TCD8 (método: Citometria de Fluxo-Facscalibur-multitest); Perfil bioquímico geral: Glicemia de jejum, HDL, Colesterol total, Triglicerídeos (método: Colorimétrico - QS), LDL, VLDL (método: Eq. De Friedwald); Transaminase Glutâmico Oxalacética - TGO/AST e Transaminase Glutâmico Pirúvica- TGP/ALT (método: Cinético de pontos múltiplos - QS).

\section{Análise do lactato sanguíneo de repouso}

A análise da concentração de lactato sanguíneo, em repouso, de cada participante/paciente, consistiu de duas coletas sanguíneas por meio de perfuração do dedo indicador (Lactímetro Accutrend Plus (Roche $^{\circledR}$ - Alemanha) e as fitas testes BM-Lactate (Roche $^{\circledR}$ - Alemanha). As coletas de repouso (período de 10 minutos) foram realizadas com os sujeitos na posição sentada antes do início do teste de 2400 $\mathrm{m}$ de Cooper e da sessão simulada de treinamento resistido. A concentração final na condição de repouso foi obtida pela média das duas coletas.

\section{Análise da cinética de lactato durante o treinamento resistido}

Para esta avaliação foi respeitado o período de duas semanas de adaptação e somente foi realizada após a conclusão do teste de 10 RM. Uma sessão simulada de treinamento resistido (uma volta no circuito, 10 repetições a $60 \%$ de carga) foi executada e as coletas sanguíneas aconteceram nos momentos anteriores à sessão simulada e ao final desta, assim como aos 15 , 30, 45, 60 e 120 minutos de repouso passivo.

\section{Análise da cinética de lactato durante o treinamento aeróbio}

A análise foi realizada durante a execução do teste de 2400 metros. As coletas aconteceram antes do teste, ao final do mesmo e aos cinco e 10 minutos de recuperação ativa e passiva, respectivamente.

\section{Avaliação da aptidão cardiorrespiratória}

Para essa avaliação foi utilizado o Teste de 2400 $m$ de Cooper, que consiste na realização de um deslocamento de 2400 metros, na máxima velocidade possível, permitindo aos participantes caminhar ou correr. Através deste teste é possível a verificação indireta do Consumo máximo de oxigênio $\left(\mathrm{VO}_{2 \operatorname{máx}}\right)$ pela fórmula $\mathrm{VO}_{2 \max }=$ distância/tempo x $0,2+3,5^{20}$.

\section{Avaliação da composição corporal}

Foi utilizado aparelho de bioimpedância elétrica (Maltron Body Composition Analyzer ${ }^{\circledR}$, Warwick) para a mensuração das seguintes variáveis: gordura absoluta $(\mathrm{kg})$, gordura relativa (\%), massa magra absoluta (kg), massa magra relativa (\%), água absoluta $(\mathrm{Lt})$, água relativa (\%) e índice de massa corporal. Os participantes foram orientados a não ingerir bebidas alcoólicas, não consumir alimentos ou bebidas que contivessem substâncias estimulantes, ingerir a quantidade mínima de dois litros de água, assim como urinar até, no máximo, 30 minutos antes do teste. No dia da avaliação, brincos, correntes e/ou anéis e tênis dos participantes foram retirados. Para a realização do teste, os participantes foram colocados em decúbito dorsal em um colchonete. Em seguida, eletrodos foram fixados na mão e no pé esquerdo dos participantes. 
Para inferir sobre o conteúdo de tecido adiposo abdominal, foi realizada a medida da circunferência da cintura por meio de fita antropométrica inelástica da marca Sanny ${ }^{\circledR}$. A circunferência da cintura foi medida a partir do menor perímetro entre a crista ilíaca e a face externa da última costela, sem compressão da pele. O ponto de referência adotado para o diagnóstico de obesidade abdominal foi de $<102 \mathrm{~cm}$ para homens e $<88 \mathrm{~cm}$ para mulheres ${ }^{21}$.

\section{Avaliação de força e resistência muscular}

Para a padronização do treinamento e análise da força, foi utilizado um teste de 10 repetiçóes máximas (10 RM). O teste de $10 \mathrm{RM}$ foi realizado nos seguintes aparelhos: meio agachamento (barra guiada), supino reto articulado, cadeira flexora, puxada alta, "leg press" $45^{\circ}$, tríceps polia, flexão plantar, desenvolvimento, rosca direta, seguindo as seguintes recomendaçóes adaptadas de Kraemer e FrY $^{22}$ : a) aquecimento de cinco a 10 repetiçóes de 40 a $60 \%$ de carga estimada, seguido de um descanso de um minuto; $b$ ) execução de três a cinco repetições com $60 \%$ de carga estimada e um descanso de três minutos; c) Incremento do peso, tentando alcançar as 10 repetiçóes máximas em três ou cinco tentativas, usando três a cinco minutos de descanso entre uma tentativa e outra. Sendo que as mudanças de carga ocorrem da seguinte maneira: realização de menos de 10 repetiçóes, a carga foi diminuída em 2 a 10\%. Se o indivíduo realizou mais de duas repetiçôes, além da pedida, a carga sofreu alteração positiva na mesma proporção. $\mathrm{O}$ valor registrado foi o encontrado na última repetição bem-sucedida. Para análise da carga nos exercícios abdominais e de flexão plantar foi realizado o teste de resistência muscular localizada (número máximo de execuçôes por minuto).

\section{Resultados}

Uma descrição geral dos pacientes/participantes contendo sexo, idade, tempo de infecção, tratamento terapêutico e o período de uso da HAART, no momento M0 encontra-se na TABELA 3.

Ao final do período de TFC houve aumento no número de células TCD4+. Os valores das células CD45+ apresentaram uma tendência ao aumento. Já em relação aos basófilos foi encontrada diminuição. Os outros parâmetros imunológicos analisados não sofreram alteração (TABELA 4).

\section{Desenho experimental}

A seguir estão descritos os diferentes momentos do período experimental e as avaliaçóes realizadas em cada um deles:

- M0: antes de iniciar o TFC; avaliaçóes: exames bioquímicos, análise do lactato sanguíneo de repouso; cinética de lactato durante o treinamento resistido; cinética de lactato durante o treinamento aeróbio; avaliação da aptidão cardiorrespiratória; avaliação da composição corporal; avaliaçãa de força e resistência muscular;

- M8: após oito semanas de TFC; avaliaçóes: análise do lactato sanguíneo de repouso; cinética de lactato durante o treinamento resistido; cinética de lactato durante o treinamento aeróbio; avaliação da aptidão cardiorrespiratória; avaliação da composição corporal; avaliaçáo de força e resistência muscular;

- M16: ao final do período experimental, após 16 semanas de TFC; avaliaçóes: exames bioquímicos, análise do lactato sanguíneo de repouso; cinética de lactato durante o treinamento resistido; cinética de lactato durante o treinamento aeróbio; avaliação da aptidão cardiorrespiratória; avaliação da composição corporal; avaliação de força e resistência muscular.

\section{Análise estatística}

Inicialmente foi testada a normalidade dos dados pelo teste de Shapiro-Wilk, sendo adotada à estatística não paramétrica. Posteriormente, foi utilizado o teste de Wilcoxon para comparar as amostras nos momentos M0 e M16. Os momentos M0, M8 e M16 foram analisados pelo teste de Friedman. Os resultados foram expressos em média \pm desvio padrão, sendo estabelecido o nível de significância de 5\% $(\mathrm{p} \leq 0,05)$.

No que se refere ao perfil bioquímico, a concentração de Glicose, HDL-c e de triglicerídeos apresentaram diferenças estatisticamente significantes após o TFC de 16 semanas (TABELA 5).

Em relação à aptidão cardiorrespiratória e força muscular, ocorreram alteraçôes significativas nas variáveis advindas do Teste de $2400 \mathrm{~m}$ de Cooper, tais como: $\mathrm{VO}_{2 \max }$ indireto, o tempo de realização do teste e a velocidade média atingida, assim como, em todos os equipamentos avaliados no teste de 10 RM (TABELA 6). 
Trombeta J CS, et al.

Os resultados referentes à composição corporal não apresentaram diferenças significativas quando comparados os três momentos avaliados (TABELA 7).

TABELA 3 - Perfil dos participantes submetidos ao treinamento físico.

Os resultados estão expressos como média \pm desvio padrão.
Os valores estão expressos em média \pm desvio padrão;

Teste de Wilcoxon;

* Para os valores de $p$

$\leq 0,05$;

$\mathrm{N}=12$.

$\frac{\text { Variáveis }}{N}$

Sexo masculino

Sexo feminino

Idade (anos)

Tempo de infecção (anos)

Tempo de uso da HAART (anos)
Números

12

3

9

$39,75 \pm 10,67$

$7,75 \pm 7,88$

$6,41 \pm 5,93$

TABELA 4 - Aspectos imunológicos dos participantes submetidos ao TFC de 16 semanas.

\begin{tabular}{lccc}
\hline & M0 & M16 & p-valor \\
\hline Carga viral (cópiasml) & $12.896 \pm 28.627$ & $9.418 \pm 19.079$ & 0,620 \\
Linfócitos TCD4+ & $519 \pm 173$ & $633 \pm 222$ & $0,034^{*}$ \\
Linfócitos TCD8+ & $988 \pm 622$ & $1076 \pm 693$ & 0,239 \\
TCD4+/TCD8+ & 0,52 & 0,59 & 0,480 \\
Linfócitos TCD45+ & $1985 \pm 809$ & $2303 \pm 1015$ & 0,071 \\
Leucócitos $\left(\mathrm{mil} / \mathrm{mm}^{3}\right)$ & $5,45 \pm 1,55$ & $5,47 \pm 1,87$ & 0,889 \\
Neutrófilos $\left(\mathrm{mil} / \mathrm{mm}^{3}\right)$ & $3,17 \pm 1$ & $3,24 \pm 1,24$ & 0,889 \\
Eosinófilos $\left(\mathrm{mil} / \mathrm{mm}^{3}\right)$ & $0,22 \pm 0,1$ & $0,21 \pm 0,17$ & 0,499 \\
Basófilos $\left(\mathrm{mil} / \mathrm{mm}^{3}\right)$ & $0,05 \pm 0,03$ & $0,03 \pm 0,02$ & $0,028^{*}$ \\
Linfócitos $\left(\mathrm{mil} / \mathrm{mm}^{3}\right)$ & $1,68 \pm 0,53$ & $1,70 \pm 0,66$ & 0,779 \\
Monócitos $\left(\mathrm{mil} / \mathrm{mm}^{3}\right)$ & $0,32 \pm 0,14$ & $0,32 \pm 0,15$ & 0,528 \\
\hline
\end{tabular}

TABELA 5 - Perfil bioquímico geral dos pacientes submetidos ao TFC de 16 semanas.

Os valores estão expressos em média \pm desvio padrão;

Teste de Wilcoxon;

*Para os valores de $p$

$\leq 0,05$;

$\mathrm{N}=12$.

\begin{tabular}{lccc}
\hline & M0 & M16 & p-valor \\
\hline Glicose em jejum (mg/dL) & $93 \pm 9,88$ & $86 \pm 6,52$ & $0,007^{*}$ \\
Colesterol total (mg/dL) & $165 \pm 40,74$ & $164 \pm 32,35$ & 1 \\
HDL-c (mg/dL) & $44 \pm 9,50$ & $49 \pm 9,43$ & $0,050^{*}$ \\
LDL-c (mg/dL) & $101 \pm 43,09$ & $95 \pm 33,29$ & 0,182 \\
VLDL-c (mg/dL) & $24 \pm 11,73$ & $20 \pm 8,08$ & 0,221 \\
Triglicerídeos (mg/dL) & $135 \pm 67,61$ & $101 \pm 39,36$ & $0,041^{*}$ \\
TGO/AST (U/L) & $25,75 \pm 4,80$ & $30,24 \pm 15,42$ & 0,157 \\
TGP/ALT (U/L) & $30 \pm 11,89$ & $28,75 \pm 22,17$ & 0,656 \\
\hline
\end{tabular}

524 • Rev Bras Educ Fís Esporte, (São Paulo) 2015 Out-Dez; 29(4):519-34 
TABELA 6 - Avaliação da aptidão cardiorrespiratória e força muscular.

\begin{tabular}{|c|c|c|c|c|}
\hline & M0 & M8 & M16 & p-valor \\
\hline \multicolumn{5}{|c|}{ Aptidão cardiorrespiratória } \\
\hline $\mathrm{VO}_{2 \operatorname{máx}}\left(\mathrm{ml} \cdot \mathrm{kg}^{-1} \cdot \mathrm{min}^{-1}\right)$ & $22,68 \pm 3,32^{\mathrm{A}}$ & $28,09 \pm 4,73^{\mathrm{B}}$ & $28,07 \pm 5,20^{\mathrm{B}}$ & $<0,001^{*}$ \\
\hline Tempo (minutos) & $25,73 \pm 4,50^{\mathrm{A}}$ & $20,16 \pm 3,70^{\mathrm{B}}$ & $20,24 \pm 3,72^{\mathrm{B}}$ & $<0,001^{*}$ \\
\hline Velocidade média $(\mathrm{m} / \mathrm{s})$ & $1,50 \pm 0,27^{\mathrm{A}}$ & $2,05 \pm 0,40^{\mathrm{B}}$ & $2,04 \pm 0,45^{\mathrm{B}}$ & $<0,001^{*}$ \\
\hline \multicolumn{5}{|l|}{ Teste de 10 RM (kg) } \\
\hline 1/2 Agachamento & $38 \pm 19^{\mathrm{A}}$ & $52 \pm 23^{B}$ & $65 \pm 26^{C}$ & $<0,001^{*}$ \\
\hline Supino reto & $20 \pm 11^{\mathrm{A}}$ & $24 \pm 11^{\mathrm{B}}$ & $28 \pm 14^{\mathrm{C}}$ & $<0,001^{*}$ \\
\hline Mesa flexora & $33 \pm 15^{\mathrm{A}}$ & $39 \pm 17^{\mathrm{B}}$ & $45 \pm 17^{\mathrm{C}}$ & $<0,001^{*}$ \\
\hline Puxada alta & $27 \pm 12^{\mathrm{A}}$ & $31 \pm 13^{\mathrm{B}}$ & $34 \pm 12^{\mathrm{C}}$ & $<0,001^{*}$ \\
\hline "Leg press" 45० & $110 \pm 48^{\mathrm{A}}$ & $150 \pm 49^{\mathrm{B}}$ & $178 \pm 60^{C}$ & $<0,001^{*}$ \\
\hline Tríceps polia & $22 \pm 9^{A}$ & $26 \pm 10^{\mathrm{B}}$ & $30 \pm 13^{\mathrm{C}}$ & $<0,001^{*}$ \\
\hline Desenvolvimento & $16 \pm 10^{\mathrm{A}}$ & $21 \pm 10^{\mathrm{B}}$ & $23 \pm 11^{\mathrm{C}}$ & $<0,001^{*}$ \\
\hline Rosca direta & $14 \pm 7^{\mathrm{A}}$ & $18 \pm 6^{\mathrm{B}}$ & $20 \pm 6^{C}$ & $<0,001^{*}$ \\
\hline
\end{tabular}

Resultados expressos em média \pm desvio padrão;

Teste de Friedman;

Nível de significância $\mathrm{p} \leq 0,05$;

Diferenças estatísticas sinalizadas pelas letras $(A \neq B \neq C)$;

$\mathrm{N}=12$.

TABELA 7 - Composição corporal de pessoas vivendo com HIV/ AIDS.

\begin{tabular}{lcccc}
\hline & M0 & M8 & M16 & p-valor \\
\hline Massa corporal total (kg) & $70 \pm 20,61$ & $69,24 \pm 19,92$ & $69,08 \pm 19,58$ & 1 \\
Massa gorda absoluta (kg) & $24,28 \pm 16,32$ & $24,27 \pm 16,13$ & $23,54 \pm 15,80$ & 0,205 \\
Massa gorda relativa (\%) & $32,60 \pm 12,50$ & $32,17 \pm 12,21$ & $31,74 \pm 12,34$ & 0,094 \\
Massa muscular absoluta (kg) & $45,73 \pm 8,68$ & $45,55 \pm 8,39$ & $45,23 \pm 8,5$ & 0,196 \\
Massa muscular relativa (\%) & $68,25 \pm 12,48$ & $67,83 \pm 12,29$ & $67,52 \pm 12,37$ & 0,196 \\
Água absoluta (Lt) & $33,48 \pm 6,34$ & $33,10 \pm 6,14$ & $33,33 \pm 6,24$ & 0,436 \\
Água relativa (\%) & $49,95 \pm 9,15$ & $49,65 \pm 8,58$ & $49,65 \pm 9,04$ & 0,336 \\
IMC (kg/m $\left.{ }^{2}\right)$ & $27,50 \pm 9,7$ & $27,22 \pm 9,5$ & $26,98 \pm 9,3$ & 0,428 \\
Circunferência de cintura & & & & 0,249 \\
Mulheres (cm) & $82,5 \pm 5,1$ & $78,5 \pm 4,4$ & $81 \pm 4,8$ & 0,761 \\
Circunferência de cintura & & & & \\
Homens $(\mathrm{cm})$ & $81,5 \pm 1$ & $79 \pm 2,3$ & & \\
\hline
\end{tabular}

Resultados expressos em média \pm desvio padrão;

Teste de Friedman; Nível de significância $\mathrm{p} \leq 0,05$; $\mathrm{N}=12$.
Quando avaliada a concentração de lactato sanguíneo em repouso, foi observado um aumento quando M0 foi comparado a M8 e M16. As diferenças estatísticas estão sinalizadas na FIGURA 1, por letras $(A \neq B)$.

Ao analisarmos a cinética de lactato sanguíneo na sessão simulada durante o treinamento físico resistido, observamos aumentos significativos nas concentraçóes nos momentos inicial, final, 15, 30, 45 minutos e 60 minutos após a sessão de exercício quando comparados os momentos M0, M8 e M16. Náo sendo encontrada diferença no momento 120 minutos (TABELA 8).

No que se refere à cinética de lactato durante o teste de $2400 \mathrm{~m}$, houve alteraçóes em todos os momentos quando comparados os três momentos da avaliação (TABELA 9). 


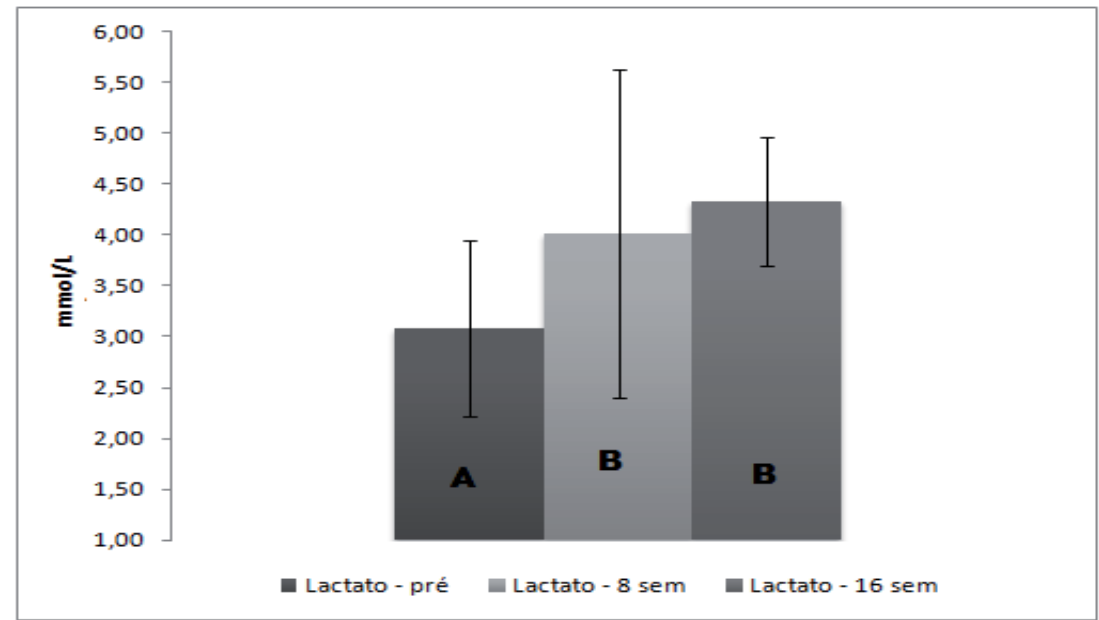

FIGURA 1 - Concentração de lactato sanguíneo de repouso dos participantes nos momentos M0, M8 e M16.

TABELA 8 - Cinética delactato sanguíneo (mmol/ L) nos momentos inicial efinal durante SSTR enos momentos de repouso.

Resultados expressos em média \pm desvio padrão;

Teste de Friedman; Nível de significância $p \leq 0,05$;

Diferenças estatísticas sinalizadas pelas letras $(A \neq B)$;

$\mathrm{N}=12$.

Resultados expressos em média desvio padrão;

Teste de Friedman;

Nível de significância $p \leq 0,05$;

Diferenças estatísticas sinalizadas pelas letras $(A \neq B)$;

$\mathrm{N}=12$.

\begin{tabular}{lcccc}
\hline & M0 & M8 & M16 & p-valor \\
\hline Inicial & $3,19 \pm 0,59^{\mathrm{A}}$ & $4,66 \pm 1,75^{\mathrm{B}}$ & $4,39 \pm 0,76^{\mathrm{B}}$ & $0,001^{*}$ \\
Final & $7,83 \pm 2,29^{\mathrm{A}}$ & $9,53 \pm 1,71^{\mathrm{B}}$ & $10,05 \pm 2,90^{\mathrm{B}}$ & $0,017^{*}$ \\
$15^{\prime}$ & $5,71 \pm 1,86^{\mathrm{A}}$ & $6,69 \pm 1,50^{\mathrm{B}}$ & $7,10 \pm 1,72^{\mathrm{B}}$ & $0,013^{*}$ \\
$30^{\prime}$ & $4,15 \pm 1,09^{\mathrm{A}}$ & $5,63 \pm 0,92^{\mathrm{B}}$ & $6,12 \pm 1,39^{\mathrm{B}}$ & $0,006^{*}$ \\
$45^{\prime}$ & $3,64 \pm 1,02^{\mathrm{A}}$ & $5,12 \pm 1,16^{\mathrm{B}}$ & $5,63 \pm 1,80^{\mathrm{B}}$ & $0,005^{*}$ \\
$60^{\prime}$ & $3,40 \pm 0,80^{\mathrm{A}}$ & $4,28 \pm 0,60^{\mathrm{B}}$ & $4,34 \pm 0,66^{\mathrm{B}}$ & $0,001^{*}$ \\
$120^{\prime}$ & $3,46 \pm 1,14$ & $4,03 \pm 0,62$ & $4,20 \pm 1,30$ & 0,107 \\
\hline
\end{tabular}

TABELA 9 - Cinética de lactato sanguíneo (mmol/ L) nos momentos inicial e final do teste de 2400 m de Cooper e nos momentos de repouso.

\begin{tabular}{lcccc}
\hline & M0 & M8 & M16 & p-valor \\
\hline Inicial & $2,9 \pm 1,10^{\mathrm{A}}$ & $3,4 \pm 1,75^{\mathrm{A}}$ & $4,3 \pm 0,76^{\mathrm{B}}$ & $0,001^{*}$ \\
Final & $4,4 \pm 3,13^{\mathrm{A}}$ & $7,5 \pm 2,40^{\mathrm{B}}$ & $8,4 \pm 2,67^{\mathrm{B}}$ & $0,001^{*}$ \\
$5^{\prime}$ & $4,25 \pm 2,96^{\mathrm{A}}$ & $5,8 \pm 1,14^{\mathrm{B}}$ & $6,7 \pm 2,44^{\mathrm{B}}$ & $0,017^{*}$ \\
$10^{\prime}$ & $4,1 \pm 2,12^{\mathrm{A}}$ & $5,5 \pm 1,74^{\mathrm{B}}$ & $5,9 \pm 1,89^{\mathrm{B}}$ & $0,017^{*}$ \\
\hline
\end{tabular}

\section{Discussão}

O presente estudo tinha como objetivos a melhora nos aspectos bioquímicos, imunológicos, cardiorrespiratório, força e composição corporal de pessoas vivendo com HIV/AIDS, assim como, na concentraçáo de lactato de repouso (e também em exercício e durante o período de recuperação; i.e., cinética) em pessoas vivendo com HIV/AIDS submetidas ao TFC de 16 semanas. O estudo contou com a participaçáo de 12 pacientes e não teve grupo controle. Ao final do TFC, houve aumento na contagem de células TCD4+ e uma tendência de aumento nas células CD45+, melhora na aptidão cardiorrespiratória 
$\left(\mathrm{VO}_{2 \operatorname{máx}}\right)$ e, ainda, melhor desempenho no teste de 10 RM. No perfil bioquímico, ocorreu melhora no perfil glicêmico, aumento nos níveis de HDL-c e diminuição nos níveis de triglicerídeos. Em relação à cinética de lactato sanguíneo, houve aumento desse substrato durante as avaliaçóes realizadas nos momentos M8 e M16 (sessóes simuladas de treinamento físico resistido e teste de $2400 \mathrm{~m}$ de Cooper), não confirmando nossa hipótese inicial para esta última variável.

Após os principais resultados serem sumarizados acima, discutimos nossos achados na forma de tópicos, devido à variedade de nossos dados e para o melhor entendimento.

\section{Sistema imunológico}

O sistema imunológico é dividido em sistema inato e adaptativo. $\mathrm{O}$ sistema inato caracteriza-se por responder aos estímulos de maneira não específica, sendo composto pelas seguintes células: neutrófilos, eosinófilos, basófilos, monócitos e células natural killer, e por fatores solúveis: sistema complemento, proteínas de fase aguda e enzimas. O sistema imune adaptativo caracteriza-se por responder ao antígeno de modo específico, apresentando memória e é composto por linfócitos $\mathrm{B}$ e $\mathrm{T}^{23-26}$. Os linfócitos $\mathrm{B}$ são células capazes de produzir anticorpos, sendo responsáveis pela imunidade humoral. Já a imunidade celular é de responsabilidade dos linfócitos T. Entre os linfócitos T, as células TCD4+ são chamadas de células auxiliares pois ajudam os linfócitos $\mathrm{B}$ e as células fagocitárias nas respostas aos antígenos; as células TCD8+ são chamadas de citotóxicas ou citolíticas, uma vez que as mesmas destroem as células infectadas por microorganismos ${ }^{23}$.

O exercício físico gera um desvio do estado de homeostase orgânica, levando à reorganização deste complexo sistema. Assim, os componentes da resposta imune, acima citados, podem sofrer modificaçóes de acordo com o estímulo recebido (estresse). A resposta aguda é a reação transitória ao estresse, enquanto o estímulo crônico gera adaptação crônica ao exercício, o qual habilita o organismo a tolerar de maneira mais adequada o estresse ${ }^{24-25,27}$. O estresse causado pelo exercício gera respostas agudas e crônicas, seja em pessoas infectadas pelo vírus HIV ou não $0^{3,6,13}$. São inúmeros os mecanismos envolvidos na modulação da resposta imunológica ao exercício $^{23,24,26}$, nấo sendo este o foco do trabalho, uma vez que seria necessária uma análise individual das células de defesa do organismo e a associaçáo das mesmas com diferentes periodizações de treinamento físico.
O sistema imunológico, associado à pratica regular de exercício físico, é uma das grandes preocupaçóes dos profissionais da saúde quando se trata de pessoas vivendo com HIV/AIDS, pois estudos demonstraram que o exercício físico de intensidade vigorosa deprime o sistema imunológico até 72 horas após a sua realização, possibilitando uma "janela aberta" ao surgimento de infecçôes. As células do sistema imune (leucócitos, neutrófilos, monócitos e linfócitos) aumentam durante e imediatamente após o exercício, sendo maiores no esforço de alta intensidade e longa duraçãa ${ }^{24,28}$.

As coletas sanguíneas para a análise do perfil imunológico foram realizadas nos momentos $\mathrm{M} 0 \mathrm{e}$ M16 (após 48 e até 72 horas da última sessão de treinamento, conforme agendamento e disponibilidade da unidade de saúde). Não realizamos as coletas de sangue para a determinação dessas células de forma aguda (imediatamente após as sessóes de treinamento físico), devido ao fato de possuir um limite para realização de exames por paciente. Não foram observadas alteraçóes em relação às células do sistema imune (leucócitos, neutrófilos, monócitos, linfócitos e eosinófilos). Somente foi observada diminuição significativa nos valores dos basófilos. A função dos basófilos está associada ao combate de alergias a longo prazo, como asma ou alergias da pele. Geralmente, essas células constituem de 0,5 a $1 \%$ do número de leucócitos do sangue humano ${ }^{29}$ e desempenha papel importante nas inflamaçôes e respostas alérgicas simples. Essas células armazenam histamina e algumas citocinas (IL-13, IL-3, IL-4) e, quando necessário, são liberadas ${ }^{30-32}$. Dessa forma, a diminuição observada não pode ser considerada negativa (por ser multifatorial) e tampouco decorrente da prática regular de exercício físico, pois, de forma hipotética, não houve a necessidade de liberação de histamina e citocinas.

Ainda se tratando de sistema imunológico, houve aumento significativo na contagem de células TCD4+ após o programa de TFC. Os estudos de Perna et al. ${ }^{33}$ e Garcia et al. ${ }^{14}$, este último realizado pelo nosso grupo de pesquisa, corroboram com nossos achados. $\mathrm{O}$ estudo de Perna et al. ${ }^{33}$ avaliou o efeito de 12 semanas de treinamento aeróbio sobre a função cardiopulmonar e contagem de células TCD4+ de três grupos vivendo com HIV/AIDS (grupo controle, grupo exercício - completou o protocolo, grupo exercício - não completou o protocolo). Além do aumento na contagem de células TCD4+ encontrado no grupo exercício, foi constatada uma diminuiçáo significativa dessas células no grupo controle e no grupo que não completou 
o protocolo de exercício. O objetivo do estudo de GARCIA et al. ${ }^{14}$ foi avaliar os efeitos do TFC de 20 semanas (três vezes na semana; 60 minutos de duração) sobre a parâmetros imunológico, cardiorrespiratório, antropométrico e bioquímicos (estresse oxidativo, dentre outras) de pessoas vivendo com HIV/AIDS; este estudo contou com a participação de 10 voluntários e seus resultados denotaram um aumento significativo na contagem das células TCD4+.

Em contrapartida, outros estudos não corroboraram este achado ${ }^{34-36}$. Sabendo que a infecção pelo vírus HIV afeta principalmente as células TCD4+ e que a progressão da doença resulta em depleção progressiva dessas células imunes, há diminuição tanto da resposta imunológica como da habilidade do organismo em combater doenças que usualmente poderia combater e, por isso, denominadas de oportunistas ${ }^{1,4}$. Nesse sentido, o treinamento físico por nós aplicado (escolhas corretas de intensidade e volume de treino) foi capaz náo só de evitar a depleção dessas células, mas, também, de aumentar o número das mesmas, como já citado. No que se refere aos mecanismos pelos quais isso ocorreu, e tendo conhecimento de que a principal função das células TCD4+ é a de transduzir sinais no momento (sinais iniciais) do reconhecimento do antígeno ${ }^{23}$, nossa hipótese, devido à inexistência de estudos que expliquem exatamente como esse fenômeno acontece, é que o TFC melhorou o funcionamento do sistema imunológico adquirido/adaptativo dos pacientes, independentemente do uso da terapia antirretroviral, sendo este um achado extremamente importante devido à vulnerabilidade dos mesmos.

No presente estudo, também foi encontrado uma tendência ao aumento das células CD45+ que, por se tratar de uma análise relativamente nova nas unidades de referência, não foram encontradas pesquisas associando as alteraçóes desse tipo de célula com o treinamento físico. De forma importante, sabe-se que a diminuição no funcionamento das células CD45+ está relacionada ao processo de envelhecimento ${ }^{37}$. Podemos sugerir que, para nossos sujeitos, o TFC, além de melhorar a função imune como já citado, também poderia ter contribuído, em partes, para a "desaceleraçáo" do processo de envelhecimento, este aumentado em pessoas vivendo com HIV/AIDS. No entanto, para confirmar essa hipótese, seria necessária a mensuração de marcadores mais fidedignos, tais como o comprimento dos telômeros e/ou a atividade da enzima telomerase; além disso, destacamos que essa nossa hipótese é meramente especulativa, uma vez que não há estudos que abordaram a CD45+ vs.
Treinamento físico vs. Processo de envelhecimento em pessoas vivendo com HIV/AIDS.

No que se refere aos valores de TCD8+, da razão TCD4+/TCD8 + e da carga viral, não houve alteraçóes. Sendo assim, podemos concluir que o programa de exercícios físicos aplicado não deprimiu o sistema imunológico dos participantes e, ainda, foi capaz de melhorar alguns de seus componentes, sendo isso de extrema importância para a saúde e para adesão nos programas das pessoas vivendo com HIV/AIDS.

\section{Perfil bioquímico}

Em relação ao perfil lipídico, houve aumento significativo na concentração de HDL-c bem como diminuição nas concentrações sanguíneas de glicose e triglicerídeos. Sabe-se que o baixo condicionamento físico, força muscular diminuída e sedentarismo aumentam a prevalência de alteraçóes metabólicas de pessoas vivendo com HIV/AIDS, principalmente se as mesmas estiverem em uso prolongado da HAART $^{38-39}$. Ao analisarmos a adesão ao programa de atividade física, observamos que, mesmo fazendo uso da medicação, o TFC foi capaz de, pelo menos em parte, melhorar os perfis glicêmico (<Glic) e lipídico (>HDL-c e <TG) dos participantes. As alteraçóes no perfil lipídico apresentam controvérsias em relação à literatura quando da aplicação do treinamento físico, pois alguns estudos mostraram resultados semelhantes ao obtidos por nós (isto é, diminuição nos valores de triglicerídeos e aumento do HDL-c ${ }^{40-41}$ e outros não (manutenção dos níveis pré e pós-treinamento físico ${ }^{42-44}$. Tais diferenças podem estar relacionadas, possivelmente, aos desenhos experimentais distintos e o local (país) de realização dos estudos. As melhoras tanto nos perfis glicêmico e lipídico, no presente estudo, podem ter ocorrido da mesma forma que em pessoas saudáveis (os mesmos benefícios), ou seja, pode ter havido melhoras na captação de glicose circulante pelo tecido muscular (aumento na expressão de GLUT4 e na fosforilação de proteínas intracelulares envolvidas nesse processo) bem como maior utilização (inclusive em repouso e não somente em exercício) dos triglicerídeos pelo músculo esquelético e, ainda, maior metabolizaçáo do mesmo pelo fígado, uma vez que, já mencionado, houve aumento nos níveis de HDL-c (lipoproteína transportadora). Dessa forma, consideramos tais respostas importantes sob o ponto de vista não somente metabólico, mas, também, no que se remete à qualidade de vida desses sujeitos, pois essas variáveis estão relacionadas, dentre outras coisas, ao risco de prejuízos ao sistema cardiovascular. 


\section{Capacidade cardiorrespiratória e força muscular}

Em indivíduos HIV+, normalmente ocorrem redução da capacidade de esforço e do $\mathrm{VO}_{2 \max }$ predito para a idade quando estes sáo comparados a indivíduos saudáveis, fazendo-se necessária a adesão e a assiduidade em programas de atividade física, a fim de minimizar tais sintomas ${ }^{13,45}$. Em nosso estudo, os indivíduos foram submetidos a 16 semanas de TFC (resistido + aeróbio) e, ao final deste, foram observados valores de $\mathrm{VO}_{2 \text { max }}$ significativamente maiores quando comparados ao período pré-treinamento (lembrando que, como limitação, não tivemos grupo controle). Além disso, foi verificada diminuição significativa no tempo de realização do teste cardiorrespiratório e, como consequência, aumento na velocidade média atingida durante este.

A melhora nesse importante parâmetro cardiorrespiratório corrobora os achados de outros autores $^{14,34,36,46}$. Outros estudos associaram o treinamento físico ao uso de fármacos como metformina e testosterona e observaram, além de alteração cardiorrespiratória, melhoras no ganho de força muscular em pessoas vivendo com HIV/AIDS ${ }^{47-48}$.

Outra alteração deletéria e comum ao organismo do indivíduo HIV+ é a redução na força muscular e a incapacidade funcional, levando a um quadro de fragilidade e dependência que pode ser associado à baixa qualidade de vida e ao aumento nos riscos de mortalidade. Nesse sentido, o treinamento físico resistido contribuiu para o aumento desta capacidade física devido às adaptaçóes musculares à carga de trabalho ${ }^{4,49}$. Em nosso trabalho, foram encontrados valores estatisticamente significativos em todos os exercícios realizados no teste de repetiçóes máximas (10 RM). Os achados obtidos por Dolan et al. ${ }^{36}$, LindEgaARD et al. ${ }^{41}$, Brito et al. ${ }^{42}$ e GARCIA et al. ${ }^{14}$ corroboram nossos achados. Há de se ressaltar tais resultados, pois as complicações associadas à infecção pelo HIV (somado ao uso prolongado da HAART), além de diminuírem a tolerância ao esforço físico, podem limitar o desempenho nas atividades da vida diária desses sujeitos. Além disso, a manutenção de valores ótimos de carga de trabalho, $\mathrm{VO}_{2 \text { máx }}$ e força pode minimizar os efeitos deletérios dos quadros de infecção oportunistas e possibilitar a manutenção ou o retorno mais rápido às funçóes diárias ${ }^{2,4,13}$. No entanto, é importante destacar que em relação ao ganho de força foi observada progressão em relaçáo aos momentos avaliados (M0, M8 e M16), fato que nâo ocorreu quando analisada a aptidão cardiorrespiratória $\left(\mathrm{VO}_{2 \max }\right)$, a qual mostrou evolução apenas quando comparados os momentos M0 e M8. Acreditamos que tal resposta pode ter ocorrido, pelo menos em parte, devido ao modo como periodizamos os treinamentos resistido e aeróbio (treinamento combinado), isto é, o treinamento resistido teve progressão de carga de exercício a cada duas semanas (TABELA 1), ao passo que no treinamento aeróbio a progressáo foi a cada quatro semanas, sofrendo alteraçōes no volume de treino (após quatro semanas), intensidade (após oito semanas) e novamente volume (após a 12 semana) (TABELA 2).

Outra possível hipótese para esses achados está relacionada à disfunção mitocondrial induzida pela HAART, uma vez que a mesma está diretamente relacionada aos distúrbios do metabolismo energético (vias aeróbia e anaeróbia) tanto à nível mitocondrial como citoplasmático, contribuindo para a uma significativa limitação nos ganhos cardiorrespiratórios dessa populaçãoo $0^{3,16,50}$. Apesar de nossos achados não apresentarem um aumento exponencial na variável aptidão cardiorrespiratória, pode-se observar a melhora em relação ao pré-treino e manutenção após as oito semanas de treinamento; tais achados são importantes devido ao fato de as complicaçóes associadas à infecção pelo HIV, assim como ao uso prolongado da HAART, diminuírem a tolerância ao esforço físico que pode limitar o desempenho nas atividades da vida diária. Sendo assim, podemos concluir que os resultados encontrados nas variáveis força e aptidáo cardiorrespiratória foram capazes de melhorar não só o desempenho físico (performance) dos participantes, mas, também, auxiliar sobremaneira na realização de atividades da vida diária, contribuindo assim, para a manutenção da saúde dos mesmos.

\section{Composição corporal}

Em nosso estudo, não foram observadas alteraçôes quando comparados os valores de composição corporal nos momentos M0, M8 e M16, corroborando os achados de Dolan et al. ${ }^{36}$. Porém, diversos estudos verificaram o contrário ${ }^{35,40,44}$. Ao analisarmos nossos resultados e, comparando-os aos trabalhos que obtiveram êxito, notamos algumas limitaçóes que poderiam explicar os valores encontrados, como por exemplo, o uso da bioimpedância em detrimento a métodos ais precisos e considerado padrôes ouro para a avaliação da composição corporal, tais como ressonância magnética, tomografia computadorizada e absorciometria com raios-x de dupla energia (DEXA). 
Vale ressaltar, ainda, que nossa amostra era forma$\mathrm{da}$, na sua maioria, por mulheres, as quais possuem menor força muscular e maior acúmulo de gordura corporal. Outro ponto elencado é a ausência de um inquérito/questionário alimentar para controle e orientação nutricional destes indivíduos. Dessa forma, o consumo tanto de calorias totais diárias quanto de micro e macronutrientes não foi acessado durante todo o período experimental. Além disso, o aumento no número de ausências, após a décima segunda semana de treinamento físico, fez com que a média de frequência dos participantes $(n=12)$, que era de $100 \%$, atingisse $85 \%$ após a $12^{\circ}$ semana de treinamento. É de suma importância destacar que os participantes que ficaram abaixo desta frequência não foram incluídos nessa pesquisa $(\mathrm{n}=4)$.

Um ponto relevante que deve ser observado é o fato de o TFC ter mantido os valores analisados nesta variável estabilizados (por exemplo: IMC, circunferência de cintura), haja visto que as presenças da obesidade, assim como o acúmulo de gordura central, são encontradas frequentemente na população avaliada. As variáveis referentes à composição corporal necessitam de constante avaliaçáo, pois as mudanças na distribuição da gordura corporal, associadas às alteraçóes metabólicas podem aumentar a morbidade e mortalidade nesta população ${ }^{51-52}$.

\section{Concentração de lactato sanguíneo (repouso, duranteSSTRetestede $2400 \mathrm{~m}$ )}

O fenômeno da hiperlactacidemia de repouso em pacientes em uso da HAART não está totalmente elucidado, mas o mesmo pode estar associado à disfunção mitocondrial causada pelo uso de ITRNs ${ }^{16-17,53}$. Em nosso estudo, observamos a ocorrência da hiperlactacidemia de repouso nos três períodos avaliados (M0, M8 e M16); nossos achados corroboram outros estudos ${ }^{16-17,50}$. No estudo de Duong et al. ${ }^{50}$, além do diagnóstico de hiperlactacidemia, os sujeitos apresentavam redução significativa na capacidade de trabalho quando comparadas às pessoas HIV + não hiperlactacidêmicas. Infelizmente, em nosso trabalho não foi possível realizar essa comparação, devido ao número reduzido de participantes (embora essa seja uma limitação clássica de estudos com pessoas vivendo com HIV/ AIDS $^{36,50}$ ) e, por essa razão, sempre corremos o risco de perda amostral durante o período experimental.

Em geral, os autores justificam o aumento do lactato sanguíneo em repouso pela falha no mecanismo da fosforilação oxidativa; este fenômeno (falha) aconteceria devido à mutação e à deterioração do DNA mitocondrial. A inibição da enzima DNA polimerase $\gamma$ altera a replicação do DNA mitocondrial (DNAmt), sendo este um codificador de subunidades de um complexo de enzimas da cadeia respiratória ${ }^{15-18,50}$.

Em nosso estudo, além de efetuarmos as análises de forma aguda e em repouso, nós as efetuamos cronicamente (M0, M8 e M16) e imediatamente após (final, 15, 30, 45, 60 e 120 min após o final das sessões) a realização de sessóes simuladas de exercícios (resistido e aeróbio). Estudos que investigaram o efeito do TFC sobre a cinética de lactato de pessoas vivendo com HIV/AIDS são escassos na literatura. O presente estudo, para nosso conhecimento, é o segundo a verificar a influência do TFC sobre a cinética de lactato de pessoas vivendo com HIV/ AIDS. O outro estudo, também realizado pelo nosso grupo de pesquisa, verificou reduçóes importantes na hiperlactacidemia de repouso e alterações na cinética de lactato sanguíneo de pessoas vivendo com HIV/ AIDS, especialmente nos momentos de recuperação ${ }^{54}$. O estudo de GARCiA et al. ${ }^{54}$ avaliou os efeitos do TFC sobre a cinética de lactato sanguíneo e a aptidão cardiorrespiratória de pacientes sujeitos com HIV. Este estudo contou a participação de 10 voluntários e consistiu de 40 min de exercícios de força seguidos de 30 min de caminhada ( 3 x/semana; duração total = 20 semanas). Cabe ressaltar que o presente trabalho e o de Garcia et al. ${ }^{54}$ não foram realizados com os mesmos sujeitos e utilizaram ergômetros distintos (pista de atletismo e esteira ergométrica, respectivamente) e, ainda, o período de TFC foi alterado de 20 para 18 semanas (incluindo neste tempo a adaptação ao treinamento), respectivamente. A mudança no período de treinamento foi uma opção para diminuir a perda amostral; desta forma, a proposta para estudos futuros é diminuir o período de treinamento para 12 semanas e assim manter um número maior de participantes até o final do período de treinamento físico. Outro ponto importante a ser observado, em relação ao teste aeróbio, é a diferença nos momentos de coleta, especialmente na recuperação. Já em relação à cinética de lactato sanguíneo após uma SSTR, não foi possível inferir algo sobre os resultados, pois o presente estudo é o primeiro a aplicar um protocolo com essas características e qualquer extrapolação dos resultados para um desfecho seria mera especulação.

Os maiores valores de lactato sanguíneo após o período de treinamento físico mostraram-se contrários à nossa hipótese inicial; no entanto, não consideramos tal fato como negativo ou prejudicial, pois esse aumento, hipoteticamente, pode ter possibilitado 
um melhor desempenho (possivelmente devido à maior formação de ATP advindo da degradação da glicose durante os testes) no teste indireto de $\mathrm{VO}_{2 \text { már }}$ e suas variáveis (velocidade e tempo) após oito e 16 semanas de treinamento, assim como em todos os exercícios de uma SSTR. Podemos sugerir, também, que os sujeitos, após adquirirem condicionamento físico adequado (como já mostrado), podem ter se tornado tolerantes à acidose e, com isso, terem obtido resultados satisfatórios. A falta de biomarcadores específicos para a elucidação de importantes questóes relacionadas a disfunção mitocondrial caracteriza-se como uma das principais limitaçóes de nosso estudo. Em estudos futuros, análises referentes aos biomarcadores específicos (ex. enzimas oxidativas [expressão e/ ou atividade], proteínas desacopladoras [UCPs], etc.) serão necessárias com o intuito de melhor elucidar esse fenômeno e, ainda, chegar ao entendimento de como a mitocôndria de pessoas vivendo com $\mathrm{HIV/}$ AIDS reage a terapia antirretroviral e, somado a isso, qual a sua real resposta ao treinamento físico.

Ao realizarmos trabalhos envolvendo análise do lactato sanguíneo, devemos considerar alguns fatores que podem influenciar sua concentração em repouso, durante e após a realização de exercícios físicos, tais como: tipo de fibra muscular, atividade glicolítica e/ou lipolítica, densidades capilar e mitocondrial e reserva de substratos energéticos ${ }^{55-56}$. O controle, mesmo que parcial, dessas variáveis, são indispensáveis para a geração de conhecimentos mais concretos acerca desse assunto tâo instigante e, ainda, obscuro do ponto de vista científico.

Podemos concluir que o TFC aplicado não prejudicou os parâmetros analisados. Notou-se uma melhora no perfil imunológico, pois não alterou a carga viral dos participantes e, ainda, foi capaz de aumentar a contagem de células TCD4+. Em relação aos parâmetros do perfil bioquímico geral, aptidão cardiorrespiratória e força muscular, observou-se melhora em variáveis importantes como: aumento nos níveis de HDL-c, consumo máximo de oxigênio, força muscular, assim como uma diminuição nos níveis de glicose e triglicerídeos, garantindo, assim, maior independência bem como melhoras da saúde e, consequentemente, da qualidade de vida dos participantes. Em relação à concentração de lactato sanguíneo nos momentos estudados, concluímos que o aumento progressivo desse substrato parece não ter sido negativo, pois, hipoteticamente, possibilitou melhor desempenho no teste indireto de $\mathrm{VO}_{2 \text { máx }} \mathrm{e}$ no aumento da força. Estudos subsequentes, a partir de parceria entre nosso grupo de estudo e outros que investigam o efeito do exercício físico sobre pessoa vivendo com HIV/AIDS, serão realizados com o objetivo de melhor elucidar essa importante e inquietante questão, pois acreditamos que um olhar mais minucioso sobre os biomarcadores mitocondriais trará respostas mais precisas e contundentes.

\begin{abstract}
Biochemical parameters and blood lactate kinetics of HIV+ individuals subject to combined physical training

The aim of the study was to verify the blood lactate kinetics (BLK) and the response to combined physical training (CPT) as well as to evaluate the effects on markers related to biochemical, immunological, cardiorespiratory and body composition in HIV + people. Twelve HIV + volunteers performed CPT during 16 weeks, and were subjected to evaluations in 3 moments: pre, 8 and 16 weeks of CPT. At the end, there was improvement in immune and cardiorespiratory parameters, muscular strength and of glycemic and lipid profiles. Concerning BLK, the concentrations were increased after the CPT. We conclude that CPT improved the measured variables, nevertheless, the issue related to the BLK requires further studies in order to clarify this occurrence.
\end{abstract}

KeY WoRdS: HIV/AIDS; Lactate kinetics; Physical aptitude; Muscle strength; Immune system. 


\section{Referências}

1. Deresz LF, Lazzarotto AR, Manfroi WC, et al. O estresse oxidativo e o exercício físico em indivíduos HIV positivos. Rev Bras Med Esporte. 2007;13:275-9.

2. Lazzarotto AR, Deresz LF, Sprinz E. HIV/AIDS e treinamento concorrente: a revisão sistemática. Rev Bras Med Esporte. 2010;16:149-53.

3. Raso V, Casseb JS, Duarte, AJ, Greve JM. Uma breve revisão sobre exercício físico e HIV/AIDS. Rev Bras Cienc Mov. 2007;15:115-26.

4. Brasil. Ministério da Saúde. Recomendaçôes para a prática de atividades físicas para vivendo com HIV e Aids. Brasília: Ministério da Saúde; 2012.

5. Junchem GM, Lazarroto AR. Treinamento físico na síndrome lipodistrófica: revisão sistemática. Rev Bras Med Esporte. 2010;16:310-3.

6. Castelo Filho A, Abrão P. Alterações metabólicas do paciente infectado por HIV. Arq Bras Endocrinol Metab. 2007;51:5-7.

7. Romancini, JL, Guariglia D, Nardo, N, Herold, P, Pimentel, GG, Pupulin, AR. Nível de atividade física e alteraçóes metabólicas em pessoas vivendo com HIV/AIDS. Rev Bras Med. 2012;18:356-60.

8. Yahiaoui A, Smith BA, Voss JG. Effect of exercise on cardiac and metabolic outcomes in people living with HIV. Annu Rev Nurs Res. 2013;31:277-96.

9. Ezema CI, Onwunali AA, Lamina S, Ezugwu UA, Amaeze AA, Nwankwo MJ. Effect of aerobic exercise training on cardiovascular parameters and CD4 cell counto f people living with human immunodeficiency virus/acquired deficiency syndrome: a randomized controlled trial. Niger J Clin Prat. 2014;17:543-48.

10. Brito CJ, Mendes EL, Bastos AA, Nobrega OT, Paula SO, Córdova C. O papel do exercício na era da terapia anti-retroviral fortemente ativa. Rev Bras de Cienc Mov. 2010;18:109-16.

11. Dutra CD, Moura AK, Saraiva DA, et al. Fatores de risco para doenças cardiovasculares em pacientes com síndrome lipodistrófica do HIV. Rev Eletrônica Gest Saúde. 2012; 3:836-51.

12. Ley C, Leach L, Barrio MR, Bassett S. Effects of an exercise programme with people living with HIV: research in disadvantaged setting. Afr J AIDS Res. 2014;13:313-19.

13. Souza FH, Marques DC. Benefícios do treinamento aeróbio e ou resistido em indivíduos HIV+: uma revisão sistemática. Rev Bras Med Esporte. 2000;15:467-71.

14. Garcia A, Fraga GA, Vieira-Junior RC, et al. Effects of combined exercise training on immunological, physical and biochemical parameters in individuals with HIV/AIDS. J Sports Sci. 2014;32:785-92.

15. Bauer AM, Sternfeld T, Horster S, Schunk M, Goebel FD, Bogner JR. Kinetics of lactate metabolism after submaximal ergometric exercise in HIV-infected patients. HIV Med. 2004;5:371-6.

16. Roge BT, Calbet JA, Moller K, et al. Skeletal muscle mitochondrial function and exercise capacity in HIV: infected patients with lipodystrophy and elevated p-lactate levels. AIDS. 2002; 16:973-82.

17. John M, Moore CB, James IR, et al. Chronic hyperlactatemia in HIV-infected patients taking antiretroviral therapy. AIDS. 2001;15:717-23.

18. Mamiafo CT, Nansseu JR, Pieme CA, Tayou C, Yonkeu JN. Hyperlactatemia in a group of HIV patients living in Yaounde-Cameroon. AIDS Res Ther. 2014;11:2-8.

19. Tanaka H, Monahan K, Seals D. Age-predicted maximal heart rate revisited. J Am Coll Cardiol. 2001;37:153-6.

20. Cooper KH. Testing and developing cardiovascular fitness within the United States Air Force. J Occup Med. 1968; 10:636-9.

21. Sociedade Brasileira de Hipertensão. Sociedade Brasileira de Cardiologia. Sociedade Brasileira de Endocrinologia e Metabologia. Sociedade Brasileira de Diabetes. Associação Brasileira para Estudos da Obesidade. I diretriz brasileira de diagnóstico e tratamento da síndrome metabólica. Rev Soc Bras Hipert. 2004;17:11-5.

22. Kraemer WJ, Fry AC. Strength testing: development and evaluation of methodology in physiological assessment oh human fitness. 2nd ed. Champaign: Human Kinetics; 1995.

23. Abbas AK, Lichtman AH, Pillai S. Imunologia: celular e molecular. 6a ed. Rio de Janeiro: Elsevier; 2008.

24. Nieman DC. Moderate exercise improves immunity and decreases illness rates. Am J Lifestyle Med. 2011;5:338-45.

25. Leandro C, Nascimento E, Castro R, Duarte JA, Castro C, Exercício físico e sistema imunológico: mecanismos e integraçóes. Rev Port Cienc Desporto. 2002;2:80-90.

26. Nieman DC. Exercise effects on systemic immunity. Immunol Cell Biol. 2000;78:496-501.

27. Rosa LFP, Vaisberg MW. Influências do exercício na resposta immune. Rev Bras Med Esporte. 2002;8:167-72. 
28. Laperriere A, Antoni M, Ironson G, et al. Effects of aerobic exercise training on lymphocyte subpopulations. Int J Sports Med.1994;15:127-30.

29. Silva EB, Grotto HZ, Vilela MM. Aspectos clínicos e o hemograma em crianças expostas ao HIV-1:comparaçáo entre pacientes infectados e soro-reversores. J Pediatr. 2001;77:503-11.

30. Merluzzi S, Betto E, Ceccaroni AA, Magris R, Giunta M, Mion F. Mast cells, basophils and B cell connection network. Mol Immunol. 2015;63:94-103.

31. Kivity S, Schwarz I, Agami O, Topilcky M, Fireman E. The effect of exercise on basophil histamine release in patients with bronchial asthma. Immunol Lett. 1994;42:1-5.

32. Knol E, Olszewski M. Basophils and mast cells: Underdog in immune regulation? Immunol Lett. 2011;138:28-31.

33. Perna FM, Laperriere A, Klimas N, et al. Cardiopulmonary and CD4 cell changes in response to exercise training in early symptomatic HIV infection. Med Sci Sports Exerc. 1999;31:973-9.

34. Rojas R, Schlicht W, Hautzinger M. Effect of exercise training on quality of life, psychological well-being, immune status, and cardiopulmonary fitness in an HIV-1 positive population. J Sport Exerc Psychol. 2003;5:440-55.

35. Engelson ES, Agin D, Kenya S, et al. Body composition and metabolic effects of a diet and exercise weight loss regimen on obese, HIV-infected women. Metabolism. 2006:55:1327-36.

36. Dolan SE, Frontera W, Librizzi J, et al. Effects of a supervised home-based aerobic and progressive resistance training regimen in women infected with human immunodeficiency virus: a randomized trial. Arch Intern Med. 2006; 166:1225-31.

37. Earl LA, Baum LG. CD45 glycosylation controls T-cell life and death. Immunology Cell Biol. 2008; 86:608-15.

38. Valente AM, Reis AF, Machado DM, Succi RC, Chacra AR. Alteraçóes da síndrome lipodistrófica do HIV. Arq Bras Endocrinol Metab. 2005;49:871-81.

39. Bacchetti P. Fat distribution in men with HIV infection. J Acquir Immune Defic Syndr. 2005;40:121-31.

40. Thöni GJ, Fredou C, Brun JF, et al. Reduction of fat accumulation and lipid disorders training in human in human immunodeficiency virus infected patients with lipodystro $\neg$ phy and/or dyslipidemia. Diabetes Metab. 2002;28:397-404.

41. Lindegaard B, Hansen T, Hvid T, et al. The effect of strength and endurance training on insulin sensitivity and fat distribution in human immunodeficiency virus-infected patients with lipodystrophy. J Clin Endocrinol Metab. 2008; 93:3860-9.

22. Brito CJ, Ferreira AA, Paula SO, Nobrega OT, Córdova C. Impacto do treinamento resistido na força e hipertrofia muscular em HIV-soropositivos. Motriz. 2013;19:313-24.

43. Terry L, Sprinz E, Stein R, Medeiros NB, Oliveira J, Ribeiro JP. Exercise training in HIV-1-infected individuals with dyslipidemia and lipodystrophy. Med Sci Sports Exerc. 2006;38:411-7.

44. Robinson FP, Quinn LT, Rimmer JH. Effects of high-intensity endurance and resistance exercise on HIV metabolic abnormalities: a pilot study. Biol Res Nurs. 2007;8:177-85.

45. Palermo PC, Feijó OG. Exercício físico e a infecção pelo HIV: atualização e recomendaçôes. Rev Bras Fisiol Exerc. 2003;2:487-99.

46 Mendes EL, Andaki AC, Amorim PR, Natali AJ, Brito, Paula SO. Treinamento físico para indivíduos HIV positivo submetidos à HAART: efeitos sobre parâmetros antropométricos e funcionais. Rev Bras Med Esporte. 2013;19:16-21.

47. Grinspoon S, Concoran C, Parlman K, Costello M, Rosentaal D, Anderson E. Effects of testosterone and progressive resistance training in eugonadal men with AIDS wasting. A randomized, controlled trial. Ann Intern Med. 2000; 133:348-55.

48. Driscoll SD, Meininger GE, Laureau MT, Dolan SE, Kililea KM, Hadigan CM. Effects of exercise training and metformin on body composition and cardiovascular indices in HIV-infected patients. AIDS. 2004;18:465-73.

49. Dudgeon WD, Phillips KD, Bopp CM, Hand GA. Physiological and psychological effects of exercise interven-tions in HIV disease. AIDS Patient Care STDS. 2004;18:81-98.

50. Duong M, Dumas JP, Buissom M, et al. Limitation of exercise capacity in nucleoside-treated HIV-infected patients with hyperlactatemia. HIV Med. 2007;8:105-11.

51. Jaime PC, Florindo AA, Latorre MR, Brasil BG, Santos EC, Segurado AA. Prevalência de sobrepeso e obesidade abdominal em indivíduos portadores de HIV/AIDS, em uso de terapia anti-retroviral de alta potência. Rev Bras Epidemiol. 2004;7:65-72.

52. Lazzaroto AR, Pereira FB, Harthmann A, Bazzo KO, Vicenzi FL, Sprinz E. Treinamento físico no risco de doença isquêmica cardíaca em sujeitos hiv/aids em uso de tarv. Rev Bras Med Esporte. 2014;20:233-36.

53. Brinkman K, Hofstede HJM, Burger DM, Smeitink JAM, Koopmans PP. Adverse effects of reverse transcriptase inhibitors: mitochondrial toxicity as common pathway. AIDS. 1998;12:1735-44. 
TrombetaJCS, et al.

54. Garcia A, Fraga GA, Silva CM, et al. Cinética de lactato de sujeitos HIV+ após 20 semanas de treinamento físico combinado. Rev Bras Ativ Fís Saúde. 2014;19:382-9.

55. Mujica I, Padilha S. Cardiorespiratory and metabolic characteristics of detraining in humans. Med Sci Sports Exerc. 2001;33:413-21.

56. Faude O, Kindermann W, Meyer T. Lactate threshold concepts. Sports Med. 2009;39:469-90.

\section{Agradecimentos}

Agradecemos o apoio financeiro da Fundação de Amparo à Pesquisa do Estado de Mato Grosso - FAPEMAT (Processo n. 331264/2012) para a realizaçáo deste projeto.

ENDEREÇO

Fabrício Voltarelli

Av. Fernando Corrêa da Costa, 2367

78060-900 - Cuiabá - MT - BRASIL

e-mail: favoltarelli@ufmt.br
Recebido para publicação: 30/08/2014

1a. revisão: 25/ 12/2014

2a. revisão: 09/01/2015

3a. revisão: 26/ 02/2015

4a. revisão: $18 / 08 / 2015$

Aceito: 21/ 09/2015 